\title{
Transcriptional targeting of virus-mediated gene transfer by the human hexokinase II promoter
}

\author{
ANN-MARIE MÄÄTTÄ ${ }^{1}$, SANNA KORJA ${ }^{1}$, HELI VENHORANTA ${ }^{1}$, TANJA HAKKARAINEN ${ }^{1}$, EIJA PIRINEN ${ }^{1}$, \\ SAMI HEIKKINEN ${ }^{1}$, RIIKKA PELLINEN $^{1}$, KIMMO MÄKINEN $^{2,3}$ and JARMO WAHLFORS ${ }^{1,2}$ \\ ${ }^{1}$ A.I. Virtanen Institute, Department of Biotechnology and Molecular Medicine, University of Kuopio; \\ ${ }^{2}$ Gene Therapy Unit, and ${ }^{3}$ Department of Surgery, Kuopio University Hospital, Kuopio, Finland
}

Received June 16, 2006; Accepted July 18, 2006

\begin{abstract}
The efficacy of the most commonly used form of suicide gene therapy, the HSV-TK/GCV method, utilizing herpes simplex virus thymidine kinase (HSV-TK) and antiviral drug ganciclovir (GCV) has been demonstrated in clinical trials. However, safer delivery of the therapeutic gene and more controlled regulation of the transgene expression, the essential prerequisites for successful therapeutic use, are still needed. We describe improved suicide gene therapy against cancer through transcripitional targeting by a strong and selective tumor-specific human hexokinase II promoter (hHKII). We examined the targeting properties of the human hHKII promoter in different human non-small cell lung cancer (NSCLC) and other human cancer cell lines using selfinactivating, VSV-G pseudotyped lentiviral vector. To confirm accurate transcriptional targeting of the hHKII promoter, the lack of transgene expression was verified in human primary bronchial epithelial and bronchial fibroblast cells. Furthermore, tissue-specific expression of the promoter was confirmed using transgenic mouse lines carrying the hHKII promoter driven luciferase reporter gene. We also tested the efficacy of the HSV-TK/GCV suicide gene therapy with the hHKII targeted lentiviral vector to NSCLC cells. Our results show that the hHKII promoter is strongly expressed in cancer cells. The targeted vector with the shortest hHKII promoter fragment (352 bp) appeared to have the best targeting properties because it efficiently governed the expression of the therapeutic gene in cancer cell lines, especially in certain non-small cell lung cancer cell lines, the transgene expression in human primary cells was virtually undetectable, and expression of the proximal hHKII promoter in transgenic mice was very low in most tissues. Also, the anti-cancer efficacy of HSV-TK/GCV therapy with the hHKII-targeted vector was comparable to that obtained with the control vector that utilized a commonly used constitutive promoter from the human elongation factor $1 \alpha$
\end{abstract}

Correspondence to: Dr Jarmo Wahlfors, A.I. Virtanen Institute, Department of Biotechnology and Molecular Medicine, P.O.Box 1627, University of Kuopio, FI-70211 Kuopio, Finland

E-mail: Jarmo.Wahlfors@uku.fi

Key words: lentivirus vector, promoter, targeting
(hEF $1 \alpha$ ) gene. In conclusion, the transcriptionally targeted lentivirus vector with hHKII promoter can successfully direct HSV-TK/GCV suicide gene therapy to non-small cell lung cancer and other tumor cell types. These results warrant further studies with orthotopic animal tumor models and primary human cancer material.

\section{Introduction}

Gene therapy as one alternative treatment form against cancer has been actively investigated during the last decades and major victories have been achieved in preclinical and clinical studies (1). From the beginning, cancer has been the number one target for gene therapy and the majority $(\sim 70 \%)$ of the current clinical gene therapy trials are aimed at treating different forms of cancer (http://www.wiley.co.uk/genetherapy/clinical/). One of the most studied approaches in cancer gene therapy is so-called suicide gene therapy that is based on prodrugactivating enzymes, which can convert harmless compounds into toxic metabolites. The 'grandfather' of all prodrugactivating gene therapies is HSV-TK/GCV gene therapy (2), a treatment that is based on introduction of the thymidine kinase gene from herpes simplex virus type 1 to tumor cells, followed by treatment with prodrug ganciclovir. Numerous preclinical experiments and clinical trials against many types of cancers have been successfully carried out using HSV-TK/ GCV therapy (3) and it has also been shown to be a potent candidate for non-small cell lung cancer (NSCLC) treatment in vitro and in vivo $(4,5)$.

The main hurdle in clinical cancer gene therapy is the difficulty to achieve efficient gene delivery into a sufficient number of tumor cells, but also the tumor specificity of current vectors is far from satisfactory. To increase the therapeutic ratio, recent studies have focused on selective killing of tumor cells, i.e. tumor targeting (6). Either controlled delivery of the therapeutic gene into the tumor tissue or controlled regulation of anti-tumor transgene expression can be used to target current cancer gene therapies. The targeting approach that allocates the gene expression to tumor cells with strong and tumor-specific promoters is called transcriptional targeting. These promoters should be highly active in tumor cells and have little or no activity in normal cells. One group of promoters with known tumor-specific features are those driving the expression of glucose-responsive genes. The expression of these genes is 
$\mathrm{hEF} 1 \alpha$

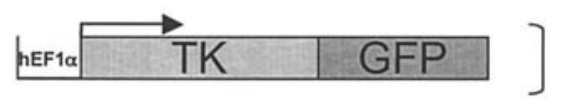

Control expression

cassette

hHKII-1820

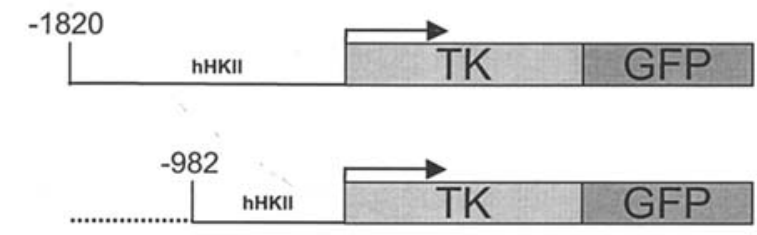

hHKII-982

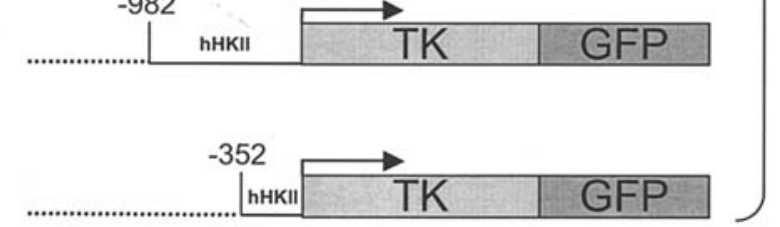

Targeted

expression

cassettes

hHKII-352

Figure 1. The expression cassettes. Schematic presentation of the control expression cassette WOX-TK-GFP with the hEF1 $\alpha$ promoter (human elongation factor $1 \alpha$ promoter) and the targeted expression cassettes hHKII-1820, hHKII-982 and hHKII-352 with different size fragments (1820, 982 and 352 bp, respectively) of the hHKII promoter. The length of the promoter fragment is indicated as base pairs to 5 ' direction from the transcription start site of the hHKII gene. All cassettes contain the fusion gene TK-GFP (herpes simplex virus thymidine kinase - green fluorescent protein). The sizes of the elements are not strictly to scale.

dependent on the microenvironment (activated glucose metabolism) of the tumor and its surroundings (7). The increased glucose metabolism in cancer cells depends to a large extent on the overexpression of hexokinase type II, the enzyme that catalyzes the first step of glycolysis (8). Cancer cells typically lack the normal sensitivity to end-product inhibition, i.e. the normal physiological feedback mechanism, whereas normal cells reduce the expression of HKII when glucose-6-phosphate is overproduced (9).

Katabi et al (10) studied the targeting properties of the rat hexokinase II (rHKII) promoter with the adenoviral vector to lung cancer cell lines. They showed that with the rHKII promoter the expression of transgene (LacZ) was high in human lung tumor cell lines compared to primary normal human cells. Encouraged by promising results from this previous study, we aimed to examine the targeting properties of the human HKII (hHKII) promoter to different cancer cell lines, especially those derived from NSCLC. We reasoned that the human promoter is more likely to function in a predicted manner and with higher activity in human malignant cells than its rodent counterparts. In addition to demonstrating the high activity of the hHKII promoter in human tumor cells in vitro and in vivo, as well as low activity in human primary cells, the activity of different promoter fragments (ranging from 352 to $1820 \mathrm{bp}$ ) was studied with cell culture experiments and by analyzing tissues of transgenic mice. Our results show the utility of the proximal hHKII-352 promoter in targeting virus vector mediated gene therapy to human NSCLC cells and other human cancer cell lines.

\section{Materials and methods}

Luciferase-assay of transgenic animals. Transgenic mouse lines (11) that express luciferase transgene under the control of 505-, 819- or 4077-bp fragments of the hHKII promoter were used to study the tissue specificity of the hHKII promoter. A Luclite luciferase reporter gene assay kit (Perkin-Elmer life sciences, Boston, MA) was used to examine hHKII promoter expression in mouse tissues in vivo. Luciferase activity was determined from tissue homogenates of four to seven transgenic mice per line. Liver samples from syngenic mice were used to obtain the background activity. Luminescence values were measured with microplate reader Microbeta (Wallac, Turku, Finland). DC protein assay (Bio-Rad, Hercules, CA) was used to determine the protein content of 1:20 diluted tissue supernatants and absorbance was measured at $750 \mathrm{~nm}$ (Labsystems Multiskan MS, Helsinki, Finland). The luciferase activity ( $\mathrm{U}=$ LCPS, light counts per second) was normalized to the protein content of the samples $(\mathrm{U} / \mu \mathrm{g})$.

\section{Promoter constructs and viral vectors}

Viral vector constructs. Schematic structures of all expression cassettes are presented in Fig. 1. All promoter fragments were excised from the full-length hHKII promoter (4077 bp) (11) using SmaI (352 bp), BlnI-XbaI (982 bp) or EcoRI-XbaI (1820 bp) digestions. The fragments were blunt-ended with Klenow polymerase and cloned into SpeI-cut, blunt-ended pWOX-TK-GFP plasmid (12). This plasmid encodes a selfinactivating, VSV-G pseudotyped lentiviral vector that carries the woodchuck hepatitis virus post-transcriptional regulatory element (WPRE) and expresses the TK-GFP gene (fusion of herpes simplex virus thymidine kinase and green fluorescent protein) (13). The control vector contained the hEF1 $\alpha$ promoter (intronless human elongation factor $1 \alpha$ ).

Virus vector preparation. VSV-G pseudotyped lentiviral vectors were produced with the standard method by transient co-transfection with $14.3 \mu \mathrm{g}$ of vector plasmid (pWOXTK-GFP, pWOX-HK1820-TK-GFP, pWOX-HK982-TKGFP or pWOX-HK352-TK-GFP), $8.6 \mu \mathrm{g}$ helper construct pCMVR8.91 and $2.1 \mu \mathrm{g}$ VSV-G envelope plasmid pMD.G (gifts from Dr Didier Trono, Swiss Institute of Technology Lausanne, Switzerland), to 293T cells (a gift from Dr Garry Nolan, Stanford University, CA). Virus titers were determined by flow cytometry (FACScalibur, Becton Dickinson, San Jose, CA, USA), measuring the number of GFP-positive 293T cells after transduction with different amounts of virus preparations (0.1-100 $\mu \mathrm{l})$. All transductions were performed in the presence of polybrene $8 \mu \mathrm{g} / \mathrm{ml}$ (hexadimethrine bromide; Sigma). The number of viral particles was determined with the p24 ELISA method (Alliance HIV-1 p24 ELISA kit, Perkin-Elmer Life Sciences) according to the manufacturer's instructions. 
Cells and culture methods

Cell lines. Eleven different tumor cell lines and two primary cell types were used in this study. Five of the cell lines represented the three main types of human NSCLC (adenocarcinoma, squamous cell carcinoma and large cell lung cancer). The rest originated from prostate, brain and ovarian cancer (two cell lines of each type). The following cell lines were obtained from ATCC (Manassas, VA): human lung adenocarcinoma cell line, A549 (ATCC CCL-185); cell lines representing human squamous cell lung cancer, SW-900 (ATCC HTB-59) and NCI-H520 (ATCC HTB-182); human large cell lung cancer cell lines, NCI-H661 (ATCC HTB-183) and NCI-H460 (ATCC HTB-177); prostate cancer cell lines, DU-145 (ATCC HTB-81) and PC-3 (ATCC CRL-1435); and brain cancer cell lines, A172 (ATCC CRL-1620) and TE671 (ATCC CCL-136). Human ovarian cancer cell line SKOV3.ipl and ovarian adenocarcinoma cell line OV-4 were kind gifts from David T. Curiel (UAB, Birmingham, AL). All cell lines were adherent and grown at $37^{\circ} \mathrm{C}$ in the presence of $5 \% \mathrm{CO}_{2}$. Cells were cultured in media recommended by ATCC, except SW-900, SKOV3.ip1 and OV-4 cells, which were grown in Dulbecco's modified Eagle's medium (DMEM) containing $10 \%$ fetal bovine serum, $2 \mathrm{mM} \mathrm{L-glutamine}$ and $50 \mu \mathrm{g} / 1$ gentamycin. The primary cell lines were purchased from Cambrex (Walkersville, MD) and cultured at $37^{\circ} \mathrm{C}$ in the presence of $5 \% \mathrm{CO}_{2}$ as follows: normal human bronchial epithelial cells (NHBE) were cultured according to the Clonetics airway epithelial cell system (BEGM BulletKit) and normal human lung fibroblast (NHFL, Clonetics) according to the Clonetics Fibroblast cell system (FGM BulletKit).

Transduction of cell lines. All viral transductions were performed on 24-well plates. The initial cell density in each well was 30,000 cells. After $24 \mathrm{~h}$, the culture medium was removed from the wells and lentiviral vectors were added in appropriate culture media containing $8 \mu \mathrm{g} / \mathrm{ml}$ polybrene. All transductions were carried out using the multiplicity of infection (MOI) 1 in $200 \mu \mathrm{l}$ volume. After $24 \mathrm{~h}, 450 \mu \mathrm{l}$ fresh medium was added onto transduced cells. Gene transfer efficiency, i.e. proportion of TK-GFP-positive cells, was determined with flow cytometry (FACScalibur, Becton Dickinson) four days after transduction. TK-GFP transgene expression level was obtained from the flow cytometer data by dividing the mean fluorescence intensity (FL1 channel) of the GFP-positive population by the mean fluorescence intensity of the GFP-negative population. The effect of different glucose concentrations on the promoter activity and gene transfer rate was investigated by growing the cells in RPMI with $10 \%$ serum supplemented with 2.0 (normal concentration), 4.5 or $7.0 \mathrm{~g} / 1$ of glucose during the period between transduction and gene transfer rate analysis, i.e. four days.

Ganciclovir sensitivity assay. Cells representing each main NSCLC type (NCI-H661, SW900 and A549) were seeded onto 6-well plates $15 \mathrm{~h}$ before transduction. Transductions were carried out for $2 \mathrm{~h}$ (targeted vector hHKII-352 and control vector $\mathrm{EF} 1 \alpha$ ), followed by addition of fresh culture medium. After three days, the transduction rate was determined by flow cytometry. Cell populations containing $2.5-20 \%$ of

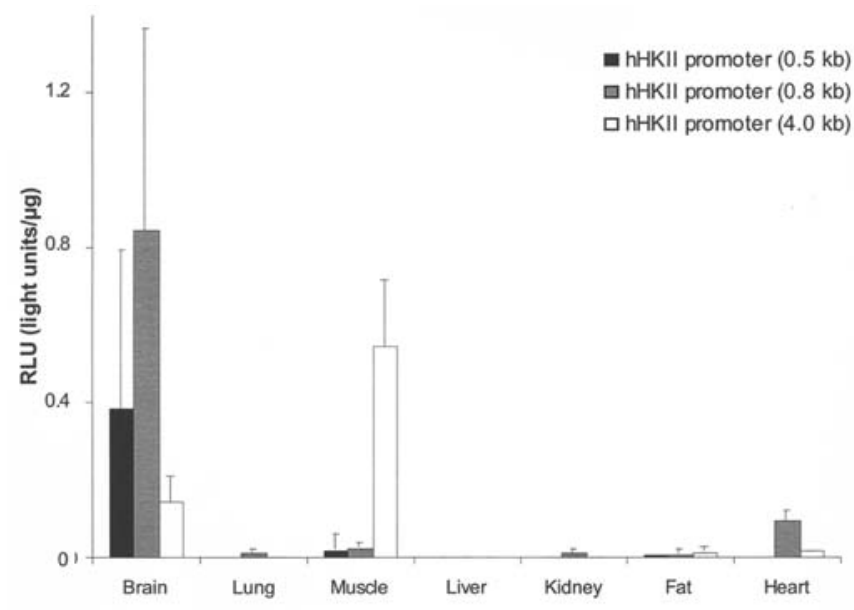

Figure 2. Analysis of hHKII promoter fragment activity in transgenic mouse tissues. Tissues were excised from three transgenic mouse lines that expressed luciferase reporter gene driven by 505-, 819- or 4077-bp fragments of the hHKII promoter $(0.5,0.8$ and $4.0 \mathrm{~kb}$, respectively). Luciferase activity was determined from tissue homogenates of 4-7 mice per line. Liver extracts of syngenic mice were used to determine the background light emission. The luciferase activity is shown as relative light units (RLU) per $\mu \mathrm{g}$ of protein, the markers indicate standard deviation.

TK-GFP-positive cells were prepared and split onto 96-well plates, 1500 cells/well. Fifteen hours later growth medium containing $10 \mu \mathrm{g} \mathrm{GCV} / \mathrm{ml}$ was added and the cells were incubated for five days in the presence of the prodrug. The viability of the cells was determined with MTT assay according to the manufacturer's instructions (Cell Proliferation Kit I, Roche Diagnostics, Indianapolis, IN).

Statistical analyses. One-way analysis of variance with Bonferroni's post hoc test for multiple comparisons was used for statistical analysis of ganciclovir sensitivity tests (program GraphPad Prism 3.0, GraphPad software, Inc., San Diego, CA).

\section{Results}

Function of hHKII-promoter in transgenic mouse tissues. To verify the activity of the human hexokinase II promoter in vivo and to determine transgene expression in different tissues, we analyzed three different transgenic mouse lines carrying the luciferase reporter gene driven by different fragments of the 5 ' flanking region of the hHKII gene (505, 819 and $4077 \mathrm{bp})$. The following lines have been previously analyzed for their transgene copy number (11): the mouse line Uku249 (505-bp promoter) harbored 8 copies; Uku268 (819-bp promoter), 2 copies; and Uku279, (4-kbp promoter) 18 copies per cells. Fig. 2 shows that hHKII activity (indicated as mean relative light units per $\mu \mathrm{g}$ of protein) was absent in the liver, low in the lung, kidney, fat and heart tissue and only moderately elevated in the brain and muscle. Interestingly, the shorter promoter fragments were the most active in the brain whereas the longest fragment provided the best promoter function in the muscle. Thus, based on the transgenic mouse data suggesting that the shorter fragments of the hHKII promoter are unlikely to provide significant activity in the lung or any other normal tissue except the brain, construction of hHKII promoter virus vectors and further in vitro experiments were warranted. 
$\mathrm{NCl}-\mathrm{H} 520$
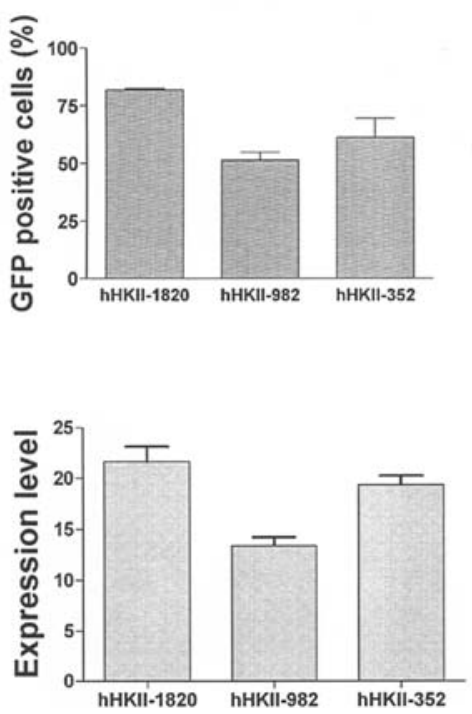

SW900
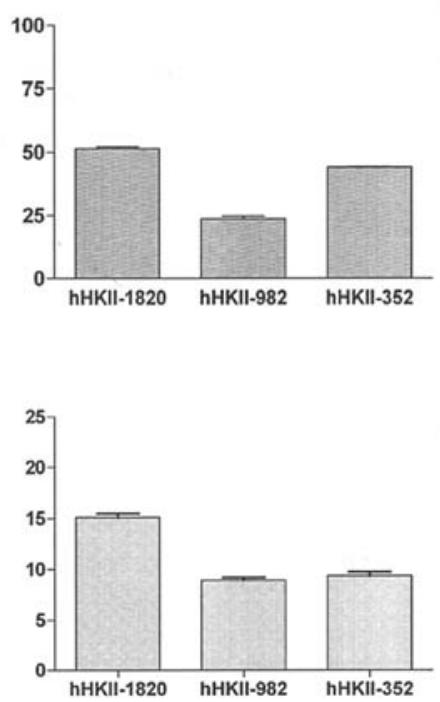

A549
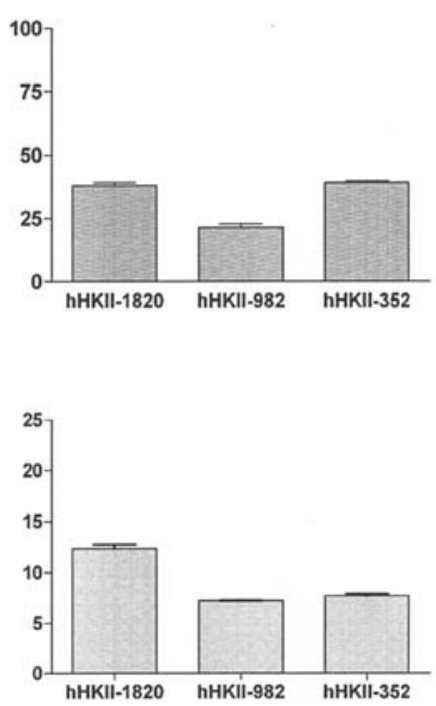

Figure 3. Comparison of different hHKII promoter fragments in human NSCLC cell lines. Transduction of three different non-small cell lung cancer lines was carried out using the targeted vectors (hHKII-1820, hHKII-982 and hHKII-352; multiplicity of infection 1). The proportion of TK-GFP-positive cells was determined with flow cytometry four days after transduction. Expression level was obtained from the flow cytometer data by dividing the mean fluorescence intensity of the GFP-positive population by the mean fluorescence intensity of the GFP-negative population. Each data point is a mean of 3 independent analyses (SD markers are indicated).

Comparison of different hHKII promoter fragments in human NSCLC cells in vitro. To examine whether the hHKII promoter possessed sufficient activity in NSCLC cells, three different fragments $(352,982$ and $1820 \mathrm{bp})$ of the hHKII promoter were cloned into the TK-GFP lentiviral vector. Vectors were produced using standard techniques as described in the Materials and methods. The titers of the vector preparations were determined with the flow cytometric method (transducing unit titer, TU/ml) and p24 ELISA method (virus protein, i.e. particle titer, $\mathrm{VP} / \mathrm{ml}$ ) to exclude the possibility that the targeted vectors would function poorly in the indicator cell line (293T) and therefore yield lower titers that would result in overestimated vector concentration. Our analyses (data not shown) revealed that all of the vector preparations contained comparable TU titers (range $4 \times 10^{5}-8 \times 10^{6} \mathrm{TU} / \mathrm{ml}$ ) and VP (range $5 \times 10^{9}-1 \times 10^{10} \mathrm{VP} / \mathrm{ml}$ ) titers and their $\mathrm{VP} / \mathrm{TU}$ ratios were within the range of 900-1100. Thus, it appeared that the targeted vectors were fully functional and yielded gene transfer and transgene expression comparable to that obtained with the control vector.

Next, the gene transfer efficiency and transgene expression level of the targeted vectors in three different NSCLC cell lines were evaluated. The purpose of this experiment was to verify the promoter function in human lung cancer cells and identify the best promoter fragment for further studies. The results showed that all three promoter fragments were functional and yielded expression of the TK-GFP gene with a low multiplicity of infection, MOI 1 (Fig. 3). Upon comparison of the vectors, it appeared that the longest 1820-bp promoter was slightly more efficient than the other two promoters in all cell lines. This also applied to the gene transfer rate and TKGFP expression level in most cases, yet the difference between the longest and the shortest promoter was not substantial.
Table I. Transduction (Td) rates and transgene expression (Exp.) levels of the control vector (promoter hEF1 $\alpha$ ) and three different targeted vectors to human primary cells (NHBE, normal human bronchial epithelial cells; NHFL, normal human fibroblast cells).

hEF1 $\alpha$ hHKII-1820a hHKII-982a hHKII-352a

\section{NHBE}

$\begin{array}{lllll}\text { Td }(\%) & 26.6 \pm 1.6 & 0 & 0 & 0\end{array}$

Exp. level $8.0 \pm 0.3$

NHFL

$\begin{array}{lrrrr}\text { Td }(\%) & 25.4 \pm 3.7 & 8.5 \pm 0.3 & 0.7 \pm 0.3 & 3.0 \pm 0.2 \\ \text { Exp. level } & 8.5 \pm 0.4 & 10.2 \pm 0.2 & 7.7 \pm 0.0 & 8.4 \pm 0.1\end{array}$

${ }^{a}$ The number refers to the length of the promoter fragment (base pairs). Values are percentage of GFP positive cells \pm SD (Td), mean fluorescence intensity of GFP signals compared to mean fluorescence of the negative population \pm SD (Exp. level).

Because the sufficient expression in tumor cells is only one aspect of targeting, the lack of function in normal human cells was also verified. The targeting properties of hHKII promoters were further analyzed by transducing normal human primary cells representing bronchial epithelium (NHBE) and lung fibroblasts (NHFL). As shown in Table I, the transduction efficiency to normal human cells was very low with the targeted hHKII vectors. The control vector yielded an approximate $25 \%$ gene transfer rate in both primary cell types, whereas none of the hHKII promoters was able to drive gene expression in bronchial epithelium and the transduction rate to 
Large cell lung cancer

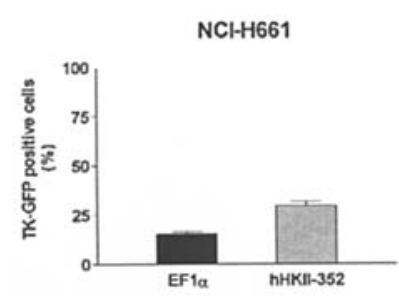

$\mathrm{NCH}+\mathrm{H} 460$

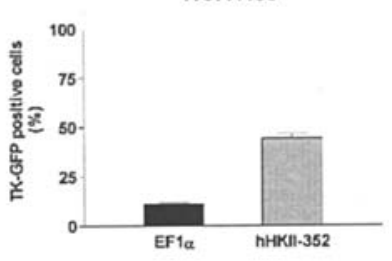

Squamous cell lung cancer

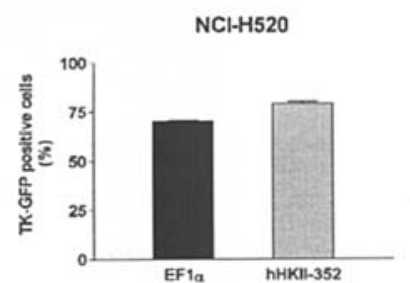

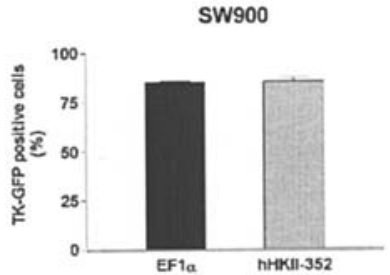

Lung adenocarcinoma

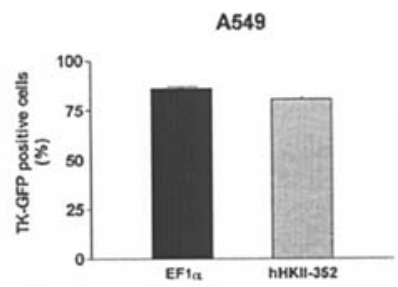

Prostate cancer
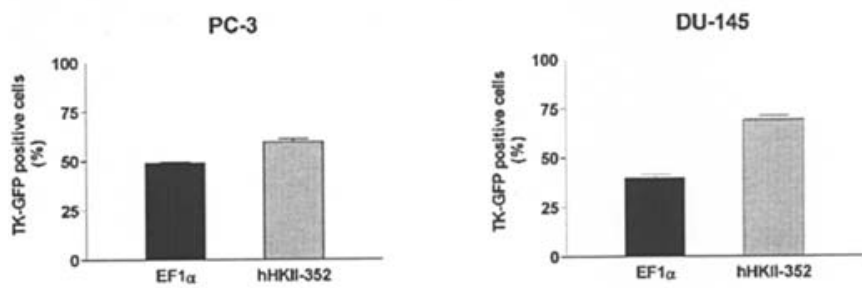

\section{Ovarian cancer}

SKOV-3 IpL.

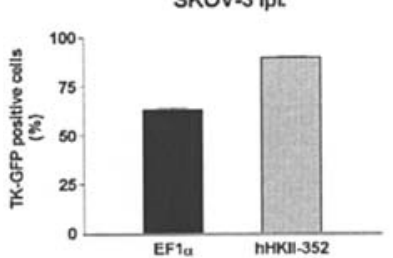

ov 4

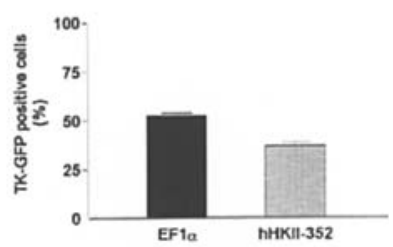

Brain cancer
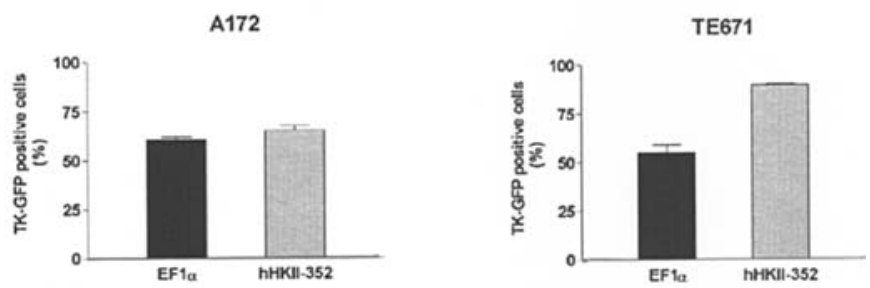

Figure 4. Transduction efficiency of lentivirus vector targeted with the hHKII-352 promoter. Transduction efficiency with the control vector (hEF1 $\alpha$ ) and the targeted vector with the shortest hHKII promoter (hHKII-352) was tested using cell lines representing different types of human cancer. Transduction was carried out using multiplicity of infection 1 and the proportion of TK-GFP-positive cells was determined by flow cytometry four days after transduction. Each data point is a mean of 3 independent analyses (SD markers are indicated).

fibroblasts was between $0.7 \%$ and $8.5 \%$. The mean transgene expression level in lung fibroblasts, however, did not reveal any major differences between the promoter fragments. The values were comparable (ranging from 7.7 to 10.2) and represented the same level as the control hEF1 $\alpha$ promoter (Table I).

Considering the importance of balance between proper function in tumor cells and lowest possible expression in normal human cells, we selected the shortest hHKII-352 fragment for further studies. This promoter was functional in all lung cancer cells, displayed no activity in NHBE cells and significantly less activity in NHFL cells than the long 1820-bp fragment. Furthermore, based on the data from transgenic mouse tissues, the shortest promoter is less likely to display activity in normal tissues.

Transduction of different human cancer cell lines with the hHKII-352 promoter. Next we evaluated the utility of the hHKII-352 vector compared to the control vector with the constitutive hEF1 $\alpha$ promoter in NSCLC cell lines (adenocarcinoma, squamous cell lung cancer and large cell lung cancer) and other malignant cell lines representing human prostate, ovarian and brain cancer. Both the control vector and the targeted vector yielded high gene transfer rates (typically between $50 \%$ and $90 \%$ of GFP-positive cells) with MOI 1 in all studied human cancer cell lines (Fig. 4). Quite surprisingly, the hHKII-352 promoter vector turned out to be equally good, or in many cases, even more efficient in transducing human tumor cells than the hEF1 $\alpha$ promoter. This control promoter, known to be universally active in many different cell and tissue types, resulted in better transduction efficiency only in the OV4 ovarian cancer cell line. Thus, the selected targeting promoter was not only virtually silent in two different primary cell types, but appeared also to be highly active in malignant cells.

We also compared the activity of both promoters by analyzing the data obtained from flow cytometry (Fig. 5). The hEF1 $\alpha$ promoter provided a somewhat higher transgene expression level in most cell lines. The hHKII-352-based expression level was 30\%-90\% of the control vector expression in $8 / 11$ cell lines. The control promoter yielded the highest expression compared to the hHKII-352 promoter in A549 lung adenocarcinoma and DU-145 prostate carcinoma cell lines. However, in two cell lines (SKOV3.ipI ovarian carcinoma and TE671 brain tumor) the targeted vector displayed 30\% and $100 \%$ higher transgene expression than the control promoter, respectively.

Because in the large cell lung cancer lines NCI-H661 and NCI-H460, the activity of hHKII-352 promoter was 
Large cell lung cancer
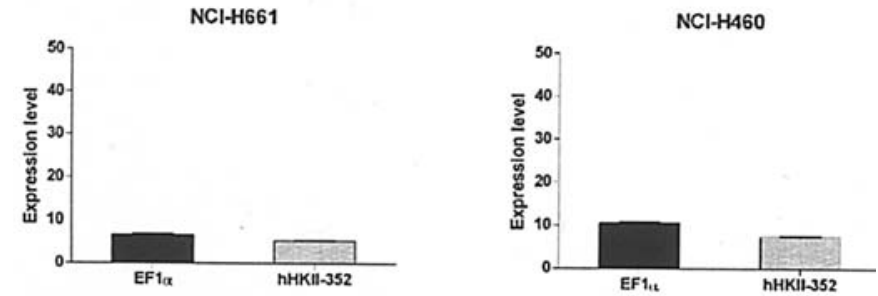

Squamous cell lung cancer
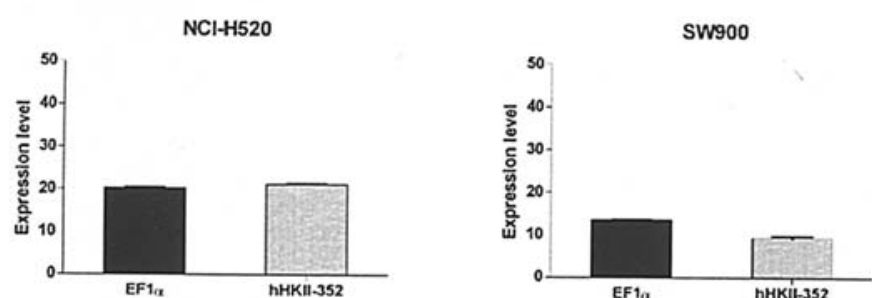

Lung adenocarcinoma

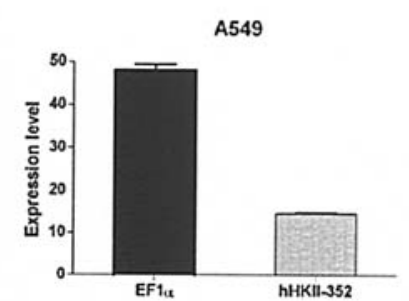

Prostate cancer
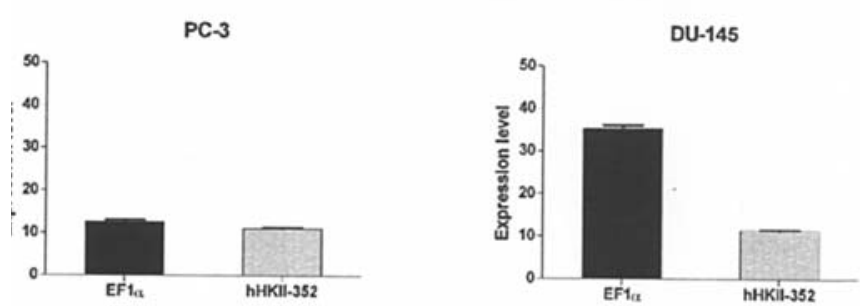

Ovarian cancer
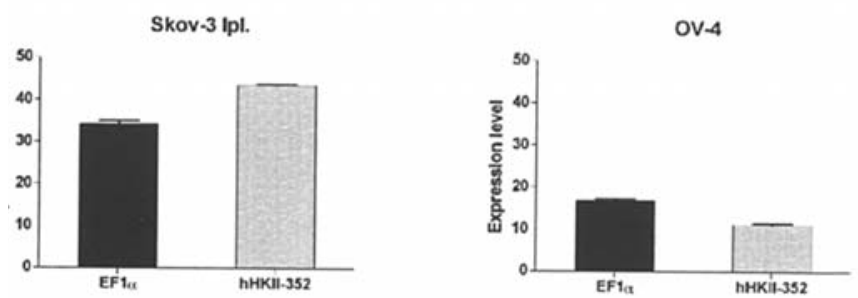

Brain cancer
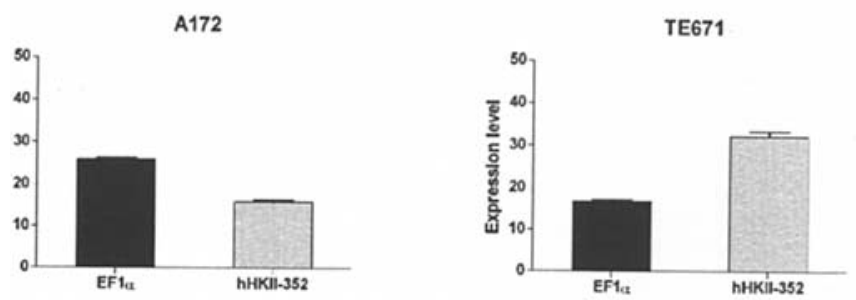

Figure 5. Level of transgene expression by the hHKII-352 promoter. Cell lines representing different types of human cancer were transduced with the control vector $(\mathrm{hEF} 1 \alpha)$ and the targeted vector with the shortest hHKII promoter (hHKII-352). Transduction was carried out using multiplicity of infection 1 and the cells were analyzed by flow cytometry four days after transduction. Expression level was obtained from the flow cytometer data by dividing the mean fluoresence intensity of the GFP-positive population by the mean fluoresence intensity of the GFP-negative population. Each data point is a mean of 3 independent analyses (SD markers are indicated).

rather low, we tried to increase its efficiency by using glucose for upregulation. When the cells were exposed to increasing glucose concentrations (from $2.0,4.5$ or $7.0 \mathrm{~g} / \mathrm{l}$ ) after transduction, no alterations in the gene expression levels were detected (data not shown). We also tested the longer promoters, but the results were identical to those obtained with the proximal promoter (data not shown), indicating that the hHKII promoter fragments in their present composition are not responsive to glucose in large cell lung cancer cell lines and cannot be markedly activated with this sugar.

HSV-TK/GCV gene therapy with hHKII-352 promoter. Finally we verified whether the chosen hHKII promoter fragment was functionally comparable to the control promoter, i.e. can it induce sufficient cytotoxic effect in a HSV-TK/GCV gene therapy setting? This was a relevant and important question since the hHKII-352 promoter provided a slightly lower gene expression level than the control promoter in NCI-H661 and SW900 cells, and a markedly lower level in A549 cells.

We transduced one cell line representing each main NSCLC type (A549, NCI-H661 and SW900) with the control vector and the targeted vector. Cell populations with $20 \%$ of TK-GFP- positive cells were prepared and subjected to GCV treatment as described in the Materials and methods. In all cell populations containing $20 \%$ of TK-GFP-positive cells, approximately $50 \%$ of the cells were killed during the exposure to $10 \mu \mathrm{g} / \mathrm{ml} \mathrm{GCV}$. Both vectors induced similar statistically significant $(\mathrm{P}<0.001)$ cytotoxicity compared to the respective control cells that were exposed to GCV. The hHKII-352 vector-transduced cells displayed comparable cell death to the $\mathrm{hEF} 1 \alpha$ vector-transduced cells. The differences of the effects of the two vectors were statistically non-significant $(\mathrm{P}>0.05)$, demonstrating that the short human hexokinase II 5 ' flanking region fragment 352 is a functionally competent promoter for targeted gene therapy of cancer (Fig. 6).

\section{Discussion}

This study was aimed at determining whether the human hexokinase II promoter is useful for transcriptional targeting of virus vectors to human tumor cells. A salient property for a tumor specific promoter is that it is functional in malignant cells. However, even more crucial is the lack of function or a very low activity in normal tissues. Since we had access to 

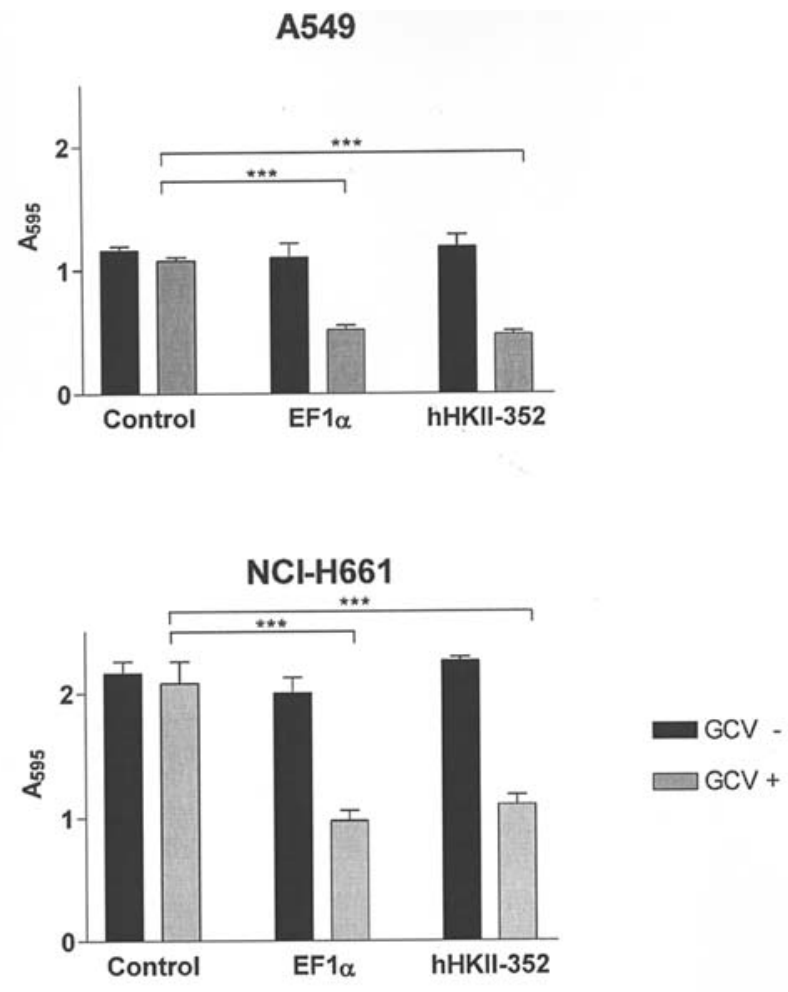

sw900

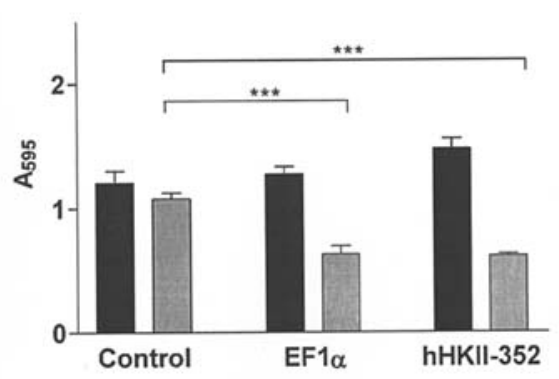

Figure 6. Ganciclovir sensitivity of human NSCLC cell lines transduced with the hHKII-352 vector. Cell lines representing adenocarcinoma (A549), large cell lung cancer (NCI-H661) and squamous cell lung cancer (SW900) were transduced with the control vector $(\mathrm{EF} 1 \alpha)$ and with the vector carrying the shortest hHKII promoter (hHKII-352). Population with $20 \%$ of TK-GFPpositive cells was prepared and incubated with $10 \mu \mathrm{g} / \mathrm{ml}$ ganciclovir for 5 days. The viability of the cells was analyzed with MTT assay (A595 values are readouts from spectrophotometer). Each data point is a mean of 3 independent analyses (SD markers are indicated). Results from statistical analysis are indicated $(* * * \mathrm{p}<0.001)$.

several mouse lines expressing luciferase under the control of fragments derived from the hHKII gene 5' flanking region (11), we analyzed the tissue samples of three different mouse lines for verification of expression in normal mouse tissues in vivo. These three lines harbored hHKII promoter fragments of 505, 819 and $4077 \mathrm{bp}$ in length and they have been shown to contain at least basal promoter activity. The present results revealed that luciferase expression was low in most tissues with all the promoter fragments, especially with the $0.5-\mathrm{kbp}$ proximal promoter (Fig. 2). The luciferase expression levels varied between different promoters and tissues, but there appeared to be no general correlation between gene copy number and transgene expression, i.e. the mouse line with the longest 4-kbp promoter harbored as much as 18 transgene copies (as opposed to 2 or 8 in the other mouse lines) but displayed the highest luciferase activity only in the muscle.

Some transgene expression was detected in typical insulinresponsive tissues, such as skeletal muscle. In normal rodent brain, hexokinase II gene expression is very low (14) while hexokinase I is highly expressed throughout development (15). This also holds true with the kidney and lung, whereas in the liver the predominant isoform is glucokinase (hexokinase IV) (16). Interestingly, however, brain tissue appeared to support the highest luciferase expression with all the hHKII promoter fragments used, confirming our previous unpublished results in the same mice, and suggesting a lack of brain-specific suppressor element(s) in these constructs. Although these hHKII promoter fragments drive some expression in the mouse brain, expression in normal human brain cells is not known. Nevertheless, our current results confirm that the proximal ( $\leq 500 \mathrm{bp}$ ) fragment of the hHKII promoter would have low activity in most tissues and qualify it as the best targeting promoter candidate for future studies.

Different hHKII promoter fragments were tested in human NSCLC cell lines (Fig. 3) and human primary cells (Table I). The short hHKII promoter turned out to be more active than the intermediate 982-bp promoter and as efficient or slightly less efficient than the long 1820-bp fragment, both in terms of gene transfer rate and transgene expression level. The reason for the somewhat higher activity of the longest promoter fragment is not known, but it is possible that the region 9821820-bp upstream from the transcription start site contains elements that are responding to stimuli present in the lung cancer cells. However, the presence of these elements is not crucial for proper function in most human tumor cells, since all the human cancer cell lines tested were excellent targets for the vector with the proximal 352-bp promoter (Fig. 4). From the viewpoint of gene therapy vector targeting, however, the lack of expression in primary bronchial epithelial cells and low level of transduction (compared to the control promoter $\mathrm{hEF} 1 \alpha$ ) in primary lung fibroblasts emphasize the utility of the short hHKII-352 promoter in vector targeting. Again, the promoter region 982-1820 bp upstream from the transcription start site appeared to harbor regulatory elements that facilitate an approximately three times higher gene transfer rate with the long hHKII-1820 fragment compared to the short 352-bp fragment in primary human fibroblasts, thus making it a less useful targeting promoter.

Comparison of the short hHKII-352 promoter with the highly active hEF1 $\alpha$ promoter revealed that the human hexokinase II core promoter was surprisingly efficient in many different human tumor cell lines (Figs. 4 and 5). We did not observe particular specificity to non-small cell lung cancer, the promoter appeared to be equally functional in all of the malignant cell lines tested. This is in line with a previous study using the rat HKII promoter (10), showing that its activity in the breast carcinoma cell line MCF-7 was comparable to the activity observed in different types of NSCLC cells. The mechanism behind the outstanding activity in tumor cells and low activity in at least two different types of human primary cells has not been elucidated, but it is likely that it is based on the higher energy consumption and consequently more 
activated glucose metabolism in most cancer types. Indeed, in many cancers, irrespective of the tissue of origin, expression of hexokinase II is greatly (>100-fold) increased by gene amplification and promoter activation to facilitate the high glycolytic rate which is needed for the maintenance of rapid growth of these cells (17-19). Thus, the elevated hHKII-352 function is probably a manifestation of aberrant promoter regulation in malignant cell types. Because the hHKII gene is upregulated by glucose in cancer (9), one would assume that the targeted hHKII vectors would be inducible by higher glucose levels in tumor cell lines. However, this appeared to be not the case and even the longest promoter fragment displayed no response to altered glucose concentrations in human large cell lung cancer cell lines. It is likely that the glucose-responsive elements of the hHKII gene are not present within the 1820-bp region upstream from the transcription start site. On the other hand, Katabi et al were able to demonstrate a 1.5-fold increase in rat HKII promoter activity with $25 \mathrm{mM}$ glucose in NCI-H661 tumor cells (10). These results, however, were obtained with a rat promoter that was markedly longer (4557 bp) than the longest human promoter fragment used in this study. Thus, it is possible that the rat $5^{\prime}$ upstream region contained at least some of the elements related to the glucose responsiveness that is displayed by the endogenous gene in cancer cells.

The final confirmation of the utility of the hHKII-352 promoter was obtained from the suicide gene therapy experiment against three different NSCLC cell lines (Fig. 6). This study revealed that hHKII-352-driven expression of TK-GFP killed all the tested tumor cell types as efficiently as hEF1 $\alpha$-based expression even though the flow cytometric analyses indicated somewhat lower activity for the targeted promoter (Fig. 5). The hHKII-352 promoter provided a mean gene expression that was $30 \%, 80 \%$ and $70 \%$ of the control promoter activity in A549, NCI-H661 and SW900 cells, respectively, demonstrating that approximately $1 / 3$ of the $\mathrm{hEF} 1 \alpha$ promoter activity is still sufficient for efficient cancer gene therapy. This was a very important finding since it is accepted that a certain threshold level of HSV-TK expression needs to be reached before the therapy is successful and the bystander effect can be achieved (20).

In conclusion we show here that the 5 ' flanking region of the hHKII gene is a suitable promoter for transcriptional targeting of virus vectors to human cancer cells. The shortest hHKII promoter fragment (352 bp) possessed favorable features for targeting; it did not display any marked activity in mouse tissues (except the brain), it governed a strong gene expression in many different human tumor cell lines but not in human primary cells, and it was able to induce a high level of cytotoxicity in a suicide gene therapy experiment against human NSCLC cells. Thus, the hHKII-352 promoter was functionally active and highly tumor-selective. These results warrant further studies with the hHKII-352-targeted vectors in animal tumor models and human primary tumor material.

\section{Acknowledgements}

This study was financially supported by the EVO-fund financing system of Kuopio University Hospital (grant no. 15204404) and Academy of Finland (grant no. 48590 to
J.W.). Research and Science Foundation of Farmos, North Savo Cultural Foundation and Kuopio University Foundation are gratefully acknowledged for supplying grants to A.-M.M.

\section{References}

1. Immonen A, Vapalahti M, Tyynela K, Hurskainen H, Sandmair A, Vanninen R, Langford G, Murray N and Yla-Herttuala S: AdvHSV-tk gene therapy with intravenous ganciclovir improves survival in human malignant glioma: a randomised, controlled study. Mol Ther 10: 967-972, 2004.

2. Moolten FL: Tumor chemosensitivity conferred by inserted herpes thymidine kinase genes: paradigm for a prospective cancer control strategy. Cancer Res 46: 5276-5281, 1986.

3. Agh M, Hochberg F and Breakefield XO: Prodrug activation enzymes in cancer gene therapy. J Gene Med 2: 148-164, 2000.

4. Hasegawa Y, Emi N, Shimokata K, Abe A, Kawabe T, Hasegawa T, Kirioka T and Saito H: Gene transfer of herpes simplex virus type I thymidine kinase gene as a drug sensitivity gene into human lung cancer cell lines using retroviral vectors. Am J Respir Cell Mol Biol 8: 655-661, 1993.

5. Maatta AM, Tenhunen A, Pasanen T, Merilainen O, Pellinen R, Makinen K, Alhava E and Wahlfors J: Non-small cell lung cancer as a target disease for herpes simplex type 1 thymidine kinase-ganciclovir gene therapy. Int J Oncol 24: 943-949, 2004.

6. Gottesman MM: Cancer gene therapy: an awkward adolescence. Cancer Gene Ther 10: 501-508, 2003.

7. Robson $\mathrm{T}$ and Hirst DG: Transcriptional targeting in cancer gene therapy. J Biomed Biotechnol 2003: 110-137, 2003.

8. Rempel A, Mathupala SP and Perdersen PL: Glucose catabolism in cancer cells: regulation of the Type II hexokinase promoter by glucose and cyclic AMP. FEBS Lett 385: 233-237, 1996.

9. Mathupala SP, Rempel A and Pedersen PL: Glucose catabolism in cancer cells. Isolation, sequence, and activity of the promoter for type II hexokinase. J Biol Chem 270: 16918-16925, 1995.

10. Katabi MM, Chan HLB, Karp SE and Batist G: Hexokinase type II: A novel tumor-specific promoter for gene-targeted therapy differentially expressed and regulated in human cancer cells. Hum Gene Ther 10: 155-164, 1999.

11. Pirinen E, Heikkinen S, Malkki M, Deeb SS, Janne J and Laakso M: Analysis of the human hexokinase II promoter in vivo: lack of insulin response within $4.0 \mathrm{~kb}$. Biochim Biophys Acta 1676: 149-154, 2004.

12. Merilainen O, Hakkarainen T, Wahlfors T, Pellinen R and Wahlfors J: HIV-1 TAT protein transduction domain mediates enhancement of enzyme prodrug cancer gene therapy in vitro: a study with TAT-TK-GFP triple fusion construct. Int J Oncol 27: 203-208, 2005.

13. Loimas S, Wahlfors $\mathrm{J}$ and Janne $\mathrm{J}$ : Herpes simplex virus thymidine kinase green fluorescent protein fusion gene: New tool for gene transfer studies and gene therapy. Biotechniques 24: 614-618, 1998.

14. Heikkinen S, Suppola S, Malkki M, Deeb SS, Janne J and Laakso M: Mouse hexokinase II gene: structure, cDNA, promoter analysis, and expression pattern. Mamm Genome 11: 91-96, 2000.

15. Griffin LD, Gelb BD, Adams V and McCabe ER: Developmental expression of hexokinase 1 in the rat. Biochim Biophys Acta 1129: 309-317, 1992.

16. Katzen HM and Schimke RT: Multiple forms of hexokinase in the rat: tissue distribution, age dependency, and properties. Proc Natl Acad Sci USA 54: 1218-1225, 1965.

17. Sebastian S and Kenkare UW: Expression of two type II-like tumor hexokinase RNA transcripts in cancer cell lines. Tumour Biol 19: 253-260, 1998.

18. Mathupala SP, Rempel A and Pedersen PL: Aberrant glycolytic metabolism of cancer cells: a remarkable coordination of genetic, transcriptional, post-translational, and mutational events that lead to a critical role for type II hexokinase. J Bioenerg Biomembr 29: 339-343, 1997.

19. Nakashima RA, Paggi MG, Scott LJ and Pedersen PL: Purification and characterization of a bindable form of mitochondrial bound hexokinase from the highly glycolytic AS-30D rat hepatoma cell line. Cancer Res 48: 913-919, 1988.

20. Drake RR, Pitlyk K, McMasters RA, Mercer KE, Young H and Moyer MP: Connexin-independent ganciclovir-mediated killing conferred on bystander effect-resistant cell lines by a herpes simplex virus-thymidine kinase-expressing colon cell line. Mol Ther 2: 515-523, 2000 